\title{
MÁS QUE UN EXPERTO. EL ROL DE JORGE ENRIQUE HARDOY EN EL DESARROLLO DE LA DISCIPLINA URBANA EN AMÉRICA LATINA
}

\author{
MORE THAN AN EXPERT. \\ JORGE ENRIQUE HARDOY'S ROLE IN THE DEVELOPMENT OF URBAN \\ DISCIPLINE IN LATIN AMERICA
}

v. 8, n. 3 [14]

set/dez (2016)

Dossiê: Cidade Latino-Americana
Alejandra Ines Monti Universidad Nacional de Rosario montialejandra@gmail.com

\section{Resumen}

Jorge Enrique Hardoy se constituye como una figura clave para comprender los procesos de transformación de la disciplina urbana en la Argentina y a escala regional entre los años 1962 y 1976. Es en su carácter de experto, pero sobre todo en su rol de promotor académico que introdujo nuevas ideas y modelos que reafirman su lugar central como transformador de una disciplina renovada bajo los preceptos de la planificación como motor para el cambio y la superación de la condición de "atraso" relativo en el proceso de modernización de los países latinoamericanos.

\section{Palabras claves}

Jorge Enrique Hardoy, Planificación Regional y Urbana, América Latina

\begin{abstract}
Jorge Enrique Hardoy is a key figure to understand the processes of transformation in urban discipline in Argentina and in the region between 1962 and 1976. His expertise, but specially his role as an academic promotor introducing new ideas and models, reaffirm his central position as a transformer in a renewed discipline under the premises of planning as a driving force for change and the overcoming of the relative "underdevelopment" in the modernization processes in Latin American countries.
\end{abstract}

\section{KeyWords}

Jorge Enrique Hardoy, Urban and Regional Planning, Latin America

\section{Introducción}

A partir de la década del cincuenta, la disciplina urbana en América Latina inicia un proceso de transformación de sus paradigmas en el marco del nuevo escenario de la posguerra caracterizado por ideal planificador como instrumento para el cambio. Así, la comprensión de la planificación como método racional, objetivo y sistemático para la toma de decisiones tendientes al desarrollo y al equilibrio territorial en clave económica, adquiere 
una renovada centralidad no sólo en términos territoriales, sino principalmente como política pública tendiente al desarrollo económico y social con el fin de superar la condición de "atraso" en Latinoamérica.

En esta línea, la trayectoria del arquitecto/planificador argentino Jorge Enrique Hardoy, entre los años 1950 y 1976, funciona como un prisma para examinar las alternativas del campo de la planificación regional y urbana tanto en la Argentina como a escala regional, ya que se consolidó como un difusor activo de esta noción de planificación que requería además un nuevo tipo de profesional que pudiese adaptarse a las nuevas condiciones -no sólo de la práctica profesional, sino principalmente de la renovación del Estado dentro del paradigma desarrollista- constituyéndose en un experto apto para integrarse a oficinas estatales, conformar equipos de trabajo y producir investigaciones y trabajos profesionales bajo la figura de la asistencia técnica al medio, lo que suponía una experticia sustentada no sólo en el campo de la práctica sino también en el académico, sumado a una mirada centrada en la técnica y en la ciencia.

No obstante, Hardoy por la relevancia y amplitud de sus actuaciones, excede el rol de experto, ya que detrás de su actividad como formador (docente y director de proyectos de investigación, consolidador de teorías y modelos a partir de la selección y edición de autores y textos), como facilitador (identificando oportunidades académicas, conformando espacios de trabajo, consolidando redes académicas y proponiendo la participación colectiva en cada una de las actividades), y como promotor (gestión y dirección de proyectos académicos, delinear vínculos institucionales a nivel local e internacional, manejar fondos y organizar reuniones científicas), es posible identificar una nueva categoría analítica que es la de "promotor académico" en el campo de la disciplina urbana ${ }^{1}$.

La importancia de J. E. Hardoy no es nueva, ya en el prólogo realizado por Marcos Kaplan para el libro Las ciudades en América Latina: seis ensayos sobre la urbanización contemporánea se destacan sus capacidades intelectuales e institucionales que le permitieron consolidarse como una figura central en el debate latinoamericano y desde allí adquirir una proyección internacional sustentada en base a su "capacidad científica, la

\footnotetext{
${ }^{1}$ En los últimos años, y desde el campo de la Sociología es posible identificar una serie de trabajos centrados en la figura de Gino Germani que abordan su actividad como constructor de la teoría sociológica en sede local a través de tres conjuntos de actividades: las vinculadas a su rol institucional (universidades y centros de investigación); su rol editorial y por último sus aportes en el campo intelectual, identificando su rol como empresario académico. Para ampliar sobre el tema ver Alejandro Blanco, Razón y Modernidad. Gino Germani y la Sociología en la Argentina; y Diego Pereyra, El desarrollo de las ciencias sociales. Tradiciones, actores e instituciones en Argentina, Chile, México y Centroamérica.
} 
teorización de alto vuelo, el agudo sentido empírico, la voluntad pragmática y el compromiso social apasionado" (Kaplan, 1972, p.14).

Veinticinco años después, y con motivo de un artículo homenaje, Ramón Gutiérrez (1995) vuelve a identificar ciertos rasgos centrales de su figura y, desde allí, esboza una serie de operaciones que abarcan desde los aportes intelectuales, la promoción de los estudios sobre historia urbana en América Latina y su capacidad de trabajo, posibilitando un acercamiento más centrado en su modo de operar que en las acciones en sí mismas.

Estas dos visiones temporalmente distante, permiten una revisión de la importancia de este actor desde una lectura centrada en lo modos de operar en el campo del urbanismo, situación que se complementa con los aportes recientes de Arturo Almandoz que posibilitan una revisión de su actividad en el marco de las acciones de los centros e institutos de investigación que operaron en el período, reconociendo las matrices que dieron forma y consolidaron en sede local la relación entre industrialización, urbanización y modernización. En esta línea se reconoce que la importancia de J. E. Hardoy no sólo se presenta a partir de la dirección de centros e instituciones, sino que es en su trabajo de redefinición de la agenda histórica de la ciudad latinoamericana donde es posible establecer su centralidad a escala continental, a partir de la operación de corrimiento teórico/metodológico desde una perspectiva evolucionista de la historia a una postura que consolida la "cultura de la planificación y la diferenciación de la historia en tanto uno de sus componentes" (Almandoz, 2006, p. 101)

Desde esta perspectiva sostenemos que el "éxito" intelectual de Hardoy puede ser explicado por la utilización eficaz de ciertas habilidades que se ajustaban perfectamente al nuevo contexto de internacionalización e institucionalización de la disciplina urbana en la posguerra, en un clima político e intelectual favorable y una dosis importante de carisma y liderazgo personal e institucional. Un liderazgo que se construyó paralelamente en múltiples espacios, lo que se corrobora tanto en los temas abordados, como en las escalas en que participa en la construcción de instituciones y redes académicas.

Una revisión de la trayectoria de J. E. Hardoy desde su actividad intelectual, institucional y editorial, permitirá iluminar ciertos mecanismos de funcionamiento de la disciplina urbana en el periodo, contribuyendo a la comprensión de los modos de construcción del conocimiento en determinadas coyunturas, sumada a la centralidad de los "expertos" en el proceso de consolidación, validación y difusión de narrativas. 


\section{Del profesional experto a la figura del promotor académico}

Desde mediados de la década del cincuenta, las universidades Argentinas transitan un clima de transformación de sus postulados en el que el dominio de la técnica y de la ciencia se constituye en un programa que cumple un rol estratégico en el desenvolvimiento económico del Estado. En esta línea la producción intelectual y científica universitaria fue fomentada tanto desde las cátedras y los nuevos institutos de investigación y asistencia técnica, como desde los organismos estatales (Consejo Nacional de Investigaciones Científicas y Técnicas, Consejo Federal de Inversiones, Consejo Nacional del Desarrollo) que a través de becas y subsidios dinaminazon la formación de posgrado en el extranjero y subsidiaron el desarrollo de proyectos de innovación teórica y técnica. Esta política pública se encontraba en estrecha relación a la necesidad de formación de recursos humanos acordes al modelo económico "desarrollista" que se pretendía aplicar en el país, modelo en el que se sustituye la universidad profesionalista por una universidad científica con un rol social y, fundamentalmente, respaldada por un discurso en pos del progreso de la ciencia y de una educación integral sustentada en una concepción universalista de la cultura.

Es en este contexto que las Ciencias Sociales comienzan a adquirir un lugar importante en las agendas de gobierno, dotando de recursos a las universidades para la financiación de proyectos y carreras científicas, posicionándolas como espacios institucionales renovados en los que se crearon centros e institutos de investigación.

Los vínculos entre política y universidad no son nuevos, pero interesa remarcar que en este período el nuevo perfil asociado a la investigación científica se proponía como un camino para modificar la realidad social y elevar los niveles de vida ${ }^{2}$, una tarea que requería de un nuevo tipo de profesional: el experto. Esta figura se considera como aquél que "posee un profundo conocimiento o es capaz de una práctica altamente calificada en un campo particular de estudio o de labor" (VILLAR, 1996). Neiburg y Plotkin, definen el rol del experto como: "(...) los técnicos, los especialistas que trabajan en y para el Estado, y más recientemente para las ONG, y los organismos internacionales. La figura del experto evoca especialización y entrenamiento académico. En su acción pública (...) actúan en nombre de la técnica y de la ciencia, reclamando hacer de la neutralidad axiológica la base para la búsqueda del bien común..."(NEIBURG y PLOTKIN, 2004, p.15).

\footnotetext{
2 Cabe destacar que esta premisa "cientificista" comienza a ser blanco de críticas a partir de mediados de la década del sesenta, situación que va a llegar al punto de mayor conflicto entre universidad y Estado con el golpe de Estado de Onganía y una nueva intervención a las universidades estatales.
}

$\begin{array}{lllll}\text { (c) Urbana: Rev. Eletrônica Cent. Interdiscip. Estud. Cid. } & \text { Campinas (SP) } & \text { v.8, n.3 [14] } & \text { p.08-29 } & \text { ISSN 1982-0569 }\end{array}$ 
Así, el dominio de la técnica y la conformación de institutos de investigación y asistencia a terceros, tuvo particular expresión en el período inaugurando un modelo alternativo de prestación de servicios respecto al profesional liberal individualista.

J. E. Hardoy a lo largo de su trayectoria encarna, de un modo muy particular, la figura del experto en la disciplina urbana, pero es a partir de la lectura de su actividad que resulta posible advertir una superación de este rol y su diversificación identificando su trabajo como el de un "constructor" de una disciplina renovada bajo los preceptos de la planificación desarrollista en clave latinoamericana: un promotor académico.

Comprender a J. E. Hardoy como "promotor académico" supone reconstruir su trayectoria, y desde allí identificar las características que posibilitan asociarlo a la capacidad para promover la producción intelectual, conformar y dirigir instituciones y centros de investigación y editar y conformar un corpus teórico de la disciplina produciendo conocimiento y generando redes académicas que supone que Hardoy fue capaz de obtener fondos para investigación, monopolizar recursos simbólicos y materiales, identificar y bloquear rivales, identificar oportunidades de investigación, negociar con sponsors y construir redes institucionales. Esta lectura enriquece la comprensión de las disputas al interior de la disciplina y del proceso de legitimación de actores e ideas en el sistema académico, a escala local y latinoamericana.

Tres factores posibilitan entender la figura de J.E. Hardoy como un promotor académico y transformador de la disciplina desde 1961 y hasta avanzada la década de los ochenta a escala latinoamericana: su producción teórica-intelectual, su rol en la creación de instituciones y centros de investigación y su labor editorial.

\section{Jorge Enrique Hardoy, promotor intelectual}

Durante el período analizado, resulta posible identificar tres estrategias de trabajo de J. E. Hardoy que lo posicionaron como un actor relevante en el contexto de una disciplina renovada bajo los preceptos de la planificación desarrollista: la actividad intelectual, la institucional y la editorial.

La producción intelectual de Hardoy en estos años se estructura a partir de dos líneas temáticas: la historia urbana y la reforma urbana. En ambos los aportes de este autor son sustantivos para la comprensión de las incorporaciones de temas y abordajes en la agenda disciplinar latinoamericana, como el caso de sus investigaciones sobre las 
ciudades precolombinas ${ }^{3}$, que inaugura la revisión histórica de la conformación del territorio latinoamericano desde una perspectiva local/regional a fin de develar las contradicciones y omisiones de la visión euro-centrista sobre los procesos evolutivos del territorio americano. Esta visión avanza en la comprensión de las lógicas (políticas, sociales, económicas y territoriales) que estructuraron las diferentes regiones del continente por un período de dos mil años, construyendo un aparato teórico-metodológico en el que la historia es presentada como un insumo para analizar la formación y consolidación del esquema urbano-territorial del continente. Esta perspectiva le permite a Hardoy identificar que los profundos desequilibrios territoriales latinoamericanos tienen sus raíces en la propia historia latinoamericana, incluso antes de la conquista española, asumiendo a la historia y las investigaciones históricas como parte del "proyecto" de desarrollo.

Desde otra línea de trabajo, los trabajos referidos a la reforma urbana se constituyeron como el segundo eje de investigación de J. E. Hardoy ${ }^{4}$, en sintonía con los debates a escala latinoamericana. En los inicios, la reforma urbana era entendida como una alternativa para la superación de los procesos de "explosión urbana" característicos del continente con la finalidad última de atacar las desigualdades a partir de un cambio en

\footnotetext{
${ }^{3}$ En la Revista A\&P N01 de 1963 se publican los primeros avances de la tesis, siendo en 1964 y en Editorial Infinito la publicación del texto completo Ciudades Precolombinas. En el mismo año, el libro fue publicado en los Estados Unidos por la editorial Walker \& Co bajo el nombre de Pre-Columbian cities, iniciando una actividad que se mantendría en el tiempo, la publicación en Norteamérica de las traducciones de los textos de habla hispana realizados por J. E. Hardoy. Las investigaciones históricas continúan siendo uno de los ejes temáticos de J. E. Hardoy durante su actividad en el CEUR, actuando entre 1966 y 1969 como director de tres investigaciones que analizan el rol de las ciudades en la historia y sus lógicas de transformación. "Recopilación de fuentes primarias sobre ciudades argentinas" junto al joven historiador Luis Alberto Romero; "Escalas y funciones urbanas en América Hispánica" y "Cartografía urbana latinoamericana. Período colonial. Recopilación y análisis". Entre 1962 y 1976, J. E. Hardoy publica catorce escritos en la temática de la historia urbana. Los mismos pueden ser agrupados en dos líneas de trabajo: la primera referida a los orígenes de los poblados americanos y su evolución en el tiempo; y la segunda, asociada a los procesos de urbanización y la planificación urbana en América Latina. En ambos casos la historia aparece como un insumo que da cuenta de los cambios físicos, económicos y sociales y de la transformación del esquema urbano/territorial definitivo. La historia aparece como reveladora de las relaciones sociales subyacentes en los cambios estructurales sobre el territorio, en una operación que intenta superar la construcción euro-centrista de una línea evolutiva necesaria para todo el universo, creando un esquema conceptual más comprensivo que, en los trabajos de J. E. Hardoy, se presenta a partir de tres enfoques diferenciados, pero que a su vez son complementarios: una clasificación en etapas evolutivas que permite definir las estructuras económicas y sociales en la historia del continente; el análisis de las configuraciones histórico culturales a fin de establecer similitudes que permitan dar cuenta de los procesos a mayor escala y en clave continental; y por último, la noción de trasplante de culturas "externas" a los espacios americanos y la aceptación de la "exterioridad" en sede local a fin de definir sus consecuencias territoriales.

${ }^{4}$ Entre 1966 y 1970 Hardoy co-dirige dos proyectos de investigación "Política sobre control y regulación del uso de la tierra urbana y suburbana" y "Mecanismos de regulación de la tierra urbana y suburbana en América del sur". El primero se enmarca dentro del contrato asumido por el CEUR con la Secretaría de Vivienda del Ministerio de Bienestar Social de la Provincia de Buenos Aires, mientras que la realización del segundo proyecto se efectúa a partir de un encargo externo, esta vez de un organismo supranacional como Naciones Unidas a través del Center for Housing, Building and Planning.
}

(c) Urbana: Rev. Eletrônica Cent. Interdiscip. Estud. Cid.

Campinas (SP)

v.8, n.3 [14]

p.08-29

ISSN 1982-0569 
la administración de la tierra y, por consiguiente, lograr una redistribución equilibrada de la urbanización y la producción en el territorio en sintonía con los principios de la política desarrollista. Sostenemos que la propuesta de Hardoy frente al tema sugiere una reforma urbana basada en la propiedad de la tierra, pero con una fuerte presencia del estado en términos de regulación con el objetivo fundamental es erradicar la especulación y el lucro exagerado.

A partir de 1971 y tras su viaje a $\mathrm{Cuba}^{5}$, Hardoy introduce modificaciones en el abordaje del tema, identificando a la reforma agraria (primera etapa del programa político revolucionario cubano) como un proceso necesario para obtener la redistribución en el territorio que permita modificar las estructuras económicas y sociales por sobre los procesos de industrialización desequilibrados. A partir de 1972 el posicionamiento de Hardoy sobre el tema va adquiriendo mayor complejización identificando a Cuba como un ejemplo de planificación a través de una nueva organización del sistema estatal y jurídico, reconociendo que la reforma urbana actúa como un instrumento estatal para la intervención en temas de vivienda y regulación del suelo y se constituye como un ejemplo para los países "sub-desarrollados". De esta forma define su postura con un posicionamiento más reformista que revolucionario, advirtiendo la necesidad de la reforma urbana como instrumento (político, económico y social) para dar respuestas a los desequilibrios y la desigualdad en el territorio, una reforma urbana a la cubana pero realizada en el marco de un gobierno democrático, sustentado en la regulación y la capitalización de plusvalías con el fin de la redistribución equilibrada, que asume a la propiedad privada como parte de una realidad que requiere limitaciones y regulaciones, pero no su desaparición, en línea con parámetros norteamericanos. Estas condiciones posibilitan comprender la centralidad asignada a la técnica des-problematizada de las posiciones ideológicas, donde el obstáculo de la transformación subyace en las estructuras políticas y no en la técnica per se, es decir la posibilidad de pensar una reforma urbana a la cubana pero sin revolución y siguiendo los parámetros norteamericanos.

\footnotetext{
${ }^{5}$ En agosto de 1970, J. E. Hardoy visita Cuba a través del programa del "Centro de Vivienda, Construcción y Planeamiento" de Naciones Unidas y el "Programa de Investigaciones de las Antillas" de la Universidad de Yale, Estados Unidos. Cuba se presenta como "el" modelo de verificación de sus ideas sobre la reforma urbana, conformando un conjunto de transformaciones estructurales a todos los niveles y en estrecha relación con un proyecto de transformación revolucionaria de la sociedad, la Revolución de 1959. Este trabajo se publica como Hardoy, J. E. y Acosta León. M: (1971) "Políticas urbanas y reforma urbana en Cuba". Caracas, Síntesis 2000. Hardoy, J. E. y Acosta León. M: (1972) "La urbanización en Cuba", en Revista de Demografía y Economía, vol 6, №1, México, Colegio de México, pp41-67
} 


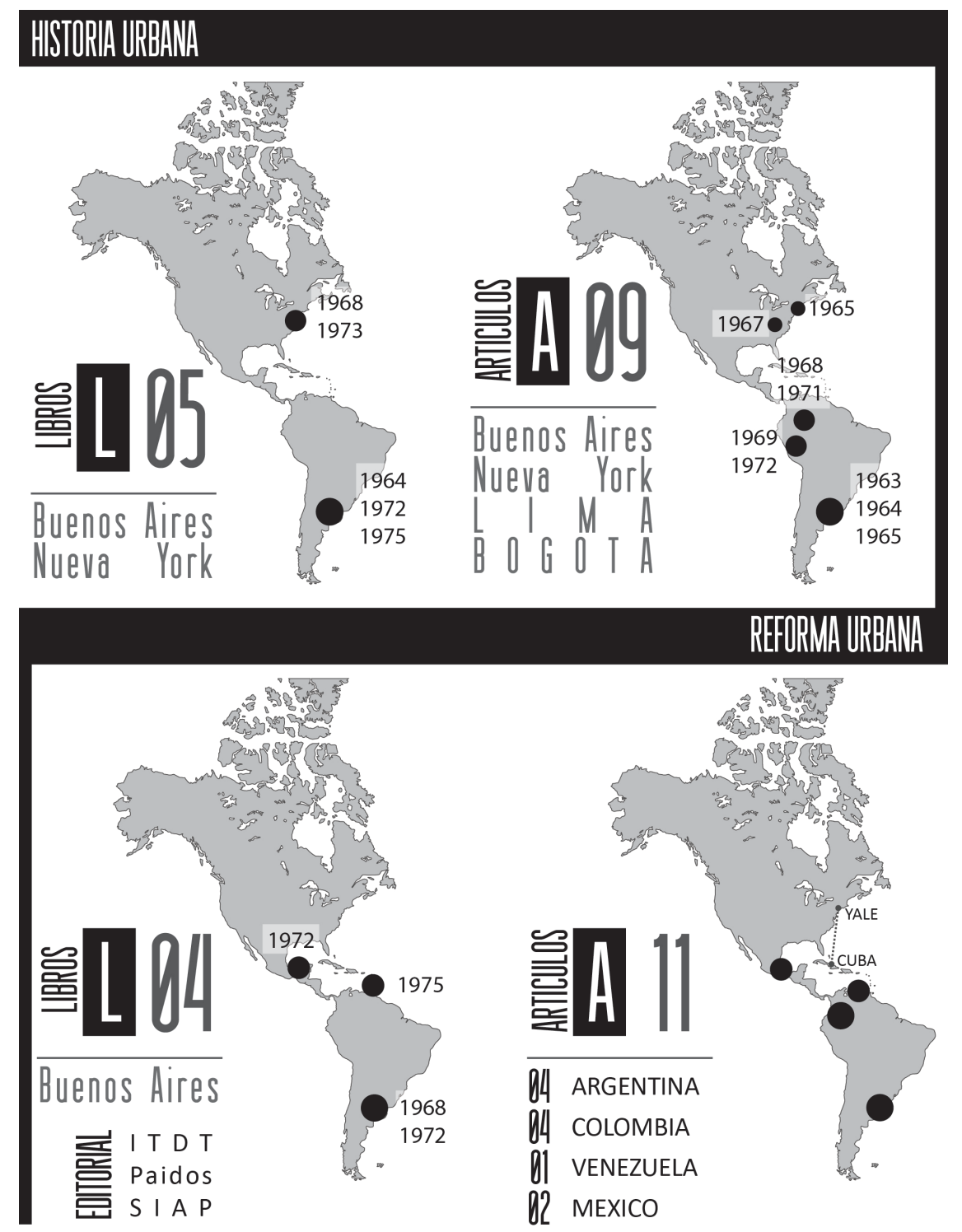

Figura 1 - Esquemas de la producción de J. E. Hardoy. Elaboración propia.

\section{Jorge Enrique Hardoy, promotor institucional}

Comprender a J. E. Hardoy como promotor institucional supone analizar su actividad en función de un conjunto de estrategias de construcción institucional, de profesionalización y de legitimación de la disciplina urbana alternativos a los vigentes en el período.

En la Argentina, Hardoy participó activamente en el Instituto de Arquitectura y Planeamiento perteneciente a la Escuela de Arquitectura con sede en la ciudad de Rosario para, en 1961, asumir el cargo de Director y delinear el nuevo Instituto de Planeamiento Regional y Urbano del Litoral (IPRUL) en línea con los postulados planteados desde la Sociedad Interamericana de Planificación (SIAP) en su Misión del año 1961. El retiro de

(c) Urbana: Rev. Eletrônica Cent. Interdiscip. Estud. Cid.

Campinas (SP)

v.8, n.3 [14]

p.08-29

ISSN 1982-0569 
Rosario (1965) y su breve paso por la Universidad de Buenos Aires (UBA - 1966) constituyen un primer período institucional vinculado a la Universidad pública Argentina que promulgaba, desde sus objetivos, la centralidad de la ciencia y la organización y promoción de unidades de investigación dependientes directamente de Rectorado. Esta apuesta educativa, en línea con la política desarrollista, se interrumpe con el golpe de estado de 1966 que no sólo clausura el sesgo científico en la UBA, sino que se constituye como un período de vaciamiento de la capacidad científica universitaria. El Equipo de Estudios Urbanos y Regionales (EEUR) no se encuentra ajeno a la experiencia, decidiendo J. E. Hardoy su incorporación como centro asociado a las filas del creciente Instituto Di Tella que, por esos años, propone la ampliación de su estructura, argumentando que "la tarea del instituto está centrada en la modernización cultural del país, con la esperanza de contribuir así a desatar el nudo cultural que traba nuestro desarrollo" (ITDT, 1966, p.5). El nuevo Centro de Estudios Urbanos y Regionales (CEUR) continua bajo la dirección de J. E. Hardoy, adecuando sus programas al accionar del ITDT y fortaleciendo los vínculos con las disciplinas sociales a partir de la congruencia de centros de investigación y actividades de integración con los otros centros ya conformados dentro del instituto.

Si bien J. E. Hardoy ejerce la dirección del CEUR por dos periodos consecutivos, su propuesta de rotación de directores se efectiviza a partir del año 1970, identificando su postura como constructor de instituciones a partir del fortaleciendo de los equipos de trabajo y su rol indiscutido como actor central del CEUR que a pesar de "ceder" la conducción del Centro, consolida desde su actuación personal en instituciones internacionales la vigencia del CEUR como nodo argentino de una red internacional, y particularmente latinoamericana, de institutos de formación e investigación en el área de la planificación regional y urbana.

En paralelo a la dirección del CEUR, en 1966 asume la presidencia de SIAP, actividad que desarrollará por dos períodos hasta el año $1970^{6}$. Allí desarrolla el proyecto editorial de SIAP, tanto a través de la revista como con la editorial, consolidando un espacio que desde los inicios marcaba la impronta de la Sociedad, que era la de convertirse en un espacio de intercambio y difusión de las actividades de sus miembros y sus respectivas instituciones.

\footnotetext{
${ }^{6}$ En el primer período lo acompaña como vice-presidente Rafael Ignacio (Pto. Rico) y mientras Carlos Zuzunaga (Perú) hace lo propio en el segundo mandato.
} 
Si en SIAP Hardoy se constituye como un actor relevante, en el Consejo Latinoamericano de Ciencias Sociales (CLACSO), es a partir de su rol como director del CEUR que participa de las primeras reuniones en 1964. Esta participación comienza a fortalecerse con la constitución de la Comisión de Desarrollo Regional y Urbano (CDRyU) en 1967, que si bien es dirigida en sus inicios por el arquitecto chileno Guillermo Geisse ${ }^{7}$, mantienen contactos frecuentes con Ricardo Jordán (CIDU) y J. E. Hardoy (CEUR), situación que se replica en la localización de sus sedes, siendo que para CLACSO es la ciudad de Buenos Aires, la coordinación de CDUyR funcionó en el CIDU, en Santiago de Chile, hecho que se modifica tras el golpe de Estado de 1973.

Las actividades durante el período 1966 y 1973 de la CDUyR se caracterizaron por el intercambio entre centros, la organización de seminarios de discusión, el desarrollo de los programas de formación, la realización de investigaciones conjuntas, la delineación de temas y metodologías de abordajes y el desarrollo de EURE como órgano difusor de las actividades de la Comisión. Para 1973, estas condiciones se modifican frente al Golpe de Estado del General Pinochet en el mes de septiembre. Si bien Geisse continua como miembro del Comité Directivo de EURE para el número de diciembre de 1973, su renuncia a la dirección del CIDU, y el posterior alejamiento de la revista, introduce modificaciones en la misma acompañado por el retiro de J. E. Hardoy del Comité Editorial frente a los cambios políticos del país. En la reunión de la Comisión de 1974, la Secretaría Ejecutiva de CLACSO designa a J. E. Hardoy como coordinador de la Comisión. Allí se acordó también la reafirmación de las líneas de investigación conformadas a partir de tres grupos de trabajo: Historia Urbana a cargo de Alejandro Moreno Toscano; Políticas Urbanas y Reforma Urbana a cargo de Oscar Moreno y Desequilibrios Regional a cargo de Fernando Travieso.

En este recorrido institucional es posible destacar algunos temas que permiten identificar el rol central de J. E. Hardoy en las instituciones que participa, no sólo por su actividad como director o coordinador, sino principalmente por la delineación de los programas de formación de posgrado, la definición de los temas y líneas de investigación, así como también las redes académicas en las que participa activamente él y sus centros.

En referencia a la enseñanza de posgrado es posible identificar un primer momento de formación de técnicos especialistas o "expertos", con diferentes grados de

\footnotetext{
${ }^{7}$ En ese período Guillermo Geisse ocupa el cargo de Jefe del Programa de Docencia del CIDU, siendo Ricardo Jordán el Director del Centro.
}

(c) Urbana: Rev. Eletrônica Cent. Interdiscip. Estud. Cid.

Campinas (SP)

v.8, n.3 [14] p.08-29

ISSN 1982-0569 
sistematización como en el IAyP o ya diseñado como programa de posgrado en el IPRUL. En el breve paso por la UBA con el EEUR el programa de formación se diluye, volviendo a funcionar en el CEUR primero como un programa de formación de técnicos y a partir de 1968 presentando una transformación y el abandono paulatino de la formación de técnicos a la formación de investigadores y teóricos, cada vez desde abordajes más alejados de la forma física, en un giro que asume la necesidad de producir teorías y modelos acordes a las necesidades locales en clave interdisciplinaria. Esto se presenta a escala latinoamericana con la diagramación del "Programa Latinoamericano de Investigación y Docencia en el campo de la urbanización" en forma conjunta con el CIDU de Chile. Y a partir de 1972 con el "Programa de Formación de Investigadores en Desarrollo Urbano y Regional" en el marco de la CDRyU de CLACSO.

En el campo de la investigación y la asistencia técnica, los cuales se constituyen como objetivos del accionar de los institutos, es posible identificar diferentes características en el tiempo, reconociendo un primer período de interrelación y retroalimentación que, con el tiempo, va adquiriendo diferentes formas y mecanismos que terminan por dividir las actividades.

En el IAyP la investigación se presentaba asociada íntegramente al trabajo de campo y la realización de Planes Reguladores, condición que a su vez se vinculaba a los programas de asistencia técnica promovidos desde el Instituto. Con el IPRUL, investigación y asistencia técnica comienzan un paulatino proceso de diferenciación, planteando la complementariedad entre las actividades de enseñanza y de investigación, mientras que en el asesoramiento técnico se consolidan los cruces entre Estado y Universidad, poniendo de manifiesto el trío compuesto por ciencia, técnica y política, que se constituye como el germen de un nuevo modelo de investigación aplicada desde la Universidad que pone en jaque el concepto de profesión liberal. En el CEUR, se abandona prácticamente la realización de Planes Reguladores y la asistencia técnica queda relegada sólo a ciertos contratos con organismos estatales u organizaciones supranacionales ${ }^{8}$, centrándose la actividad en las tareas investigación desde tres aspectos: sociales, económicos-territoriales

\footnotetext{
${ }^{8}$ Entre los trabajos realizados se encuentran "Política sobre control y regulación del uso de la tierra urbana y suburbana", en el marco del contrato asumido con la Secretaría de Vivienda del Ministerio de Bienestar Social; "Mecanismos de regulación de la tierra urbana y suburbana en América del sur", a partir de un encargo externo de las Naciones Unidas; "Diagnóstico de equipamiento comunitario y servicios públicos en áreas urbanas. Plan de acción para los sectores de población de bajos recursos", a partir del convenio con la Secretaría de Estado de Promoción y Asistencia Comunitaria del Ministerio de Bienestar Social de la Nación; "Bienestar Social e Infraestructuras de Balcarce", mediante un convenio con el partido bonaerense de Balcarce, y "Elaboración de un anteproyecto de organización institucional y jurídica para el área metropolitana de Rosario", a través de un contrato con la Provincia de Santa Fe.
} 
y los trabajos avocados a las problemáticas territoriales, que con el correr de los años se va a orientar íntegramente a la elaboración de marcos, modelos y teorías que permitan elaborar desde una postura local una base teórica propia.

El último de los ejes de trabajo de los institutos y centros se orientó a las tareas de divulgación las cuales presentan diferentes características en el tiempo, identificando nuevos modelos de institucionalización, de intercambio y difusión. Si en un primer período vinculado al IAyP, la difusión se orienta al público en general, en el IPRUL los esfuerzos están puestos en la organización de una biblioteca especializada. Con la incorporación del CEUR al ITDT, las actividades de difusión se transforman. La creación de la editorial del Instituto, permite la publicación de trabajos, investigaciones y artículos producidos en el seno del Centro. En referencia al intercambio, este último periodo se caracteriza por la ampliación de la participación en congresos y reuniones nacionales e internacionales de los actores, sumado al rol de J. E. Hardoy en instituciones interamericanas, demostrando que más allá de ejercer un cargo de dirección la presencia de Hardoy y su rol como promotor definen las líneas de acción institucional, operando en redes latinoamericanas e internacionales, participando en unidades académicas norteamericanas, así como también su actividad como coordinador de publicaciones que tienen al tema de la ciudad y sus procesos de transformación como ejes.

El golpe de Estado de 1976 en la Argentina, inauguró un período de debilitamiento de las instituciones políticas, económicas y educativas en el país. Los regímenes dictatoriales de América del Sur, la crisis del "modelo" desarrollista de la década anterior, el traspaso de una economía de cuño "proteccionista" al liberalismo y el endeudamiento progresivo y su consecuente ajuste del crecimiento interno, promovieron un cambio de perspectivas sobre las posibilidades de la modernización en América Latina, no sólo desde la mirada del Primer Mundo, sino también, a partir de los intelectuales locales y sus revisiones y puesta en crisis de la teoría de la dependencia y el par centro-periferia. 
Este contexto de transformación tuvo su correlato en las actividades del CEUR. La disminución progresiva de las actividades de investigación, sumado al exilio de sus principales figuras fue, progresivamente, desarticulando una estructura institucional que se había mantenido (con sus variaciones) por un período de quince años y qué, en cierto modo, clausuró la idea de la planificación como vehículo para el cambio.

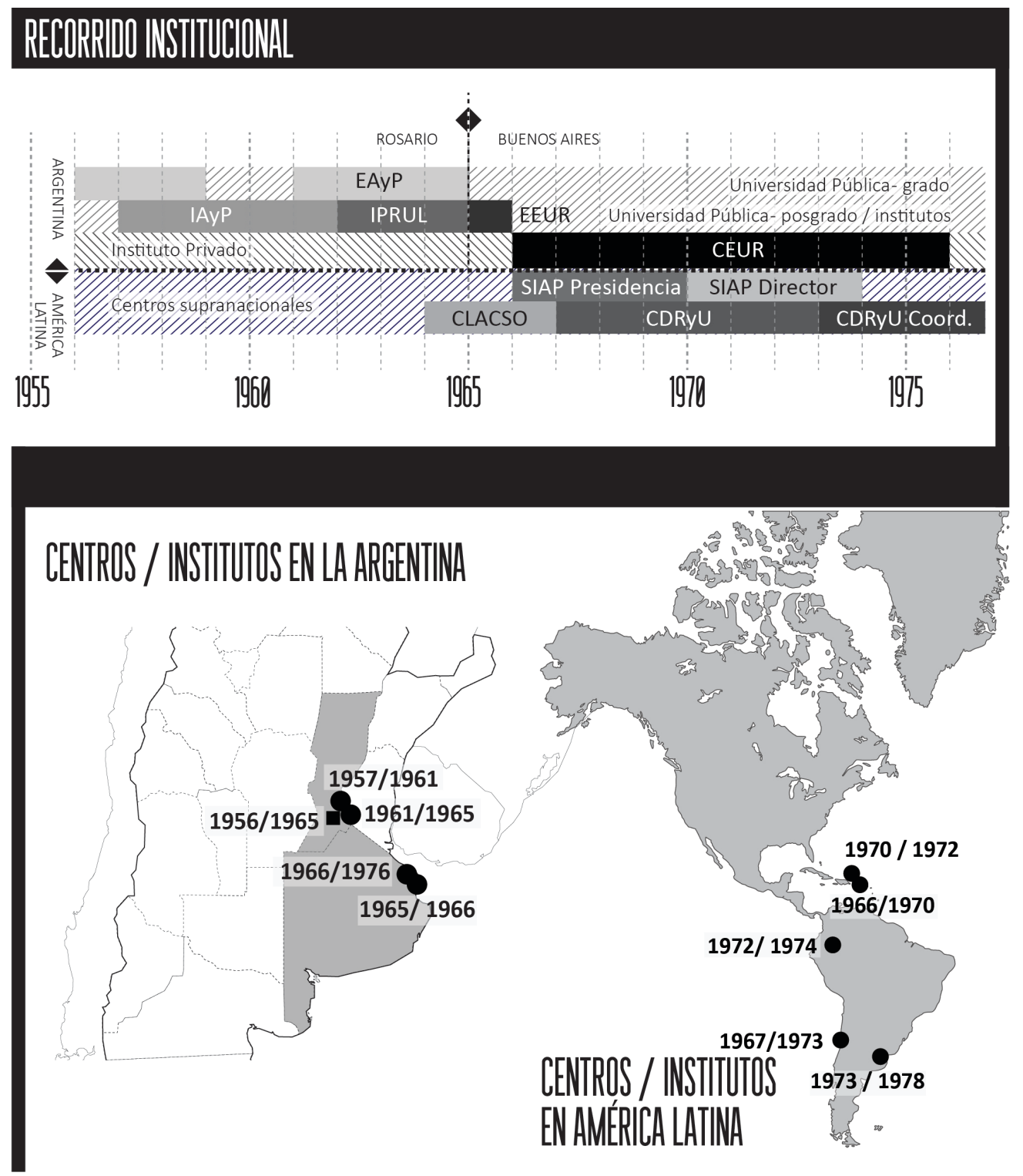

Figura 2 - Esquemas del recorrido institucional de J. E. Hardoy. Elaboración propia.

J. E. Hardoy no se encontró ajeno a las persecuciones del gobierno militar argentino, siendo que en el mes de mayo, y ante los preparativos de su estancia en Canadá 
con motivo de su participación en el Congreso Mundial del Hábitat ${ }^{9}$, este actor fue retenido por un breve lapso, situación que generó un gran impacto en la comunidad de planificadores a escala internacional que, como en el caso del director del Princeton Institute for Advanced Study, comunicaron a la Embajada de los Estados Unidos en la Argentina un pedido de informe sobre las condiciones del arresto y su pedido de excarcelación. Su liberación, agilizó su viaje a Canadá y su posterior radicación en Inglaterra junto a su familia ejerciendo como profesor visitante del Institute of Development Studies en la Universidad de Sussex y del University College en Londres por un período de dos años.

\section{Jorge Enrique Hardoy promotor editorial}

La actividad editorial de J. E. Hardoy en estos años se desarrolló a partir de la fundación del proyecto Ediciones Infinito (1954) y la participación activa como director o editor en revistas especializadas tanto en la Argentina como a escala Latinoamericana.

Un primer período de esta actividad se desarrolló entre 1954 y 1965 , momento en el que Hardoy conforma la Biblioteca de Planeamiento y Vivienda en el marco de Infinito y participa como co-director de la Revista $A \& P^{10}$. Aquí es posible destacar los intentos por consolidar una disciplina bajo los nuevos preceptos de la planificación en clave cepalina, introduciendo modificaciones en la selección de textos y autores, sumado a la incorporación de artículos centrados en la planificación regional y urbana.

Esta condición se manifiesta en la Biblioteca de Planeamiento y Vivienda a partir de dos etapas diferenciadas, la primera (1958 y 1962) caracterizada todavía por una selección heterogénea de autores y referentes que cristalizan un momento de transformación de la disciplina urbana sin una cabal diferenciación entre el Urbanismo y el Planeamiento ${ }^{11}$; y la segunda a partir de 1962 donde se introduce un cambio de referentes

\footnotetext{
${ }^{9}$ En el marco de la Conferencia de Naciones Unidas sobre Asentamientos Humanos. Allí fue elegido como Senior Fellow del International Development Research Centre de Canadá durante el período 1976/77

10 La revista A\&P. Revista de Arquitectura y Planeamiento, es editada por la Escuela de Arquitectura y Planeamiento de Rosario en el marco institucional de la Universidad Nacional del Litoral. La misma fue codirigida por Jorge Enrique Hardoy, Aníbal Moline e Iván Hernández Larguia. Se publican ocho (8) números entre 1963 y 1967, adquiriendo difusión nacional a través de la distribución de EUDEBA, en un contexto signado por el desarrollo de proyectos editoriales signados por la experimentación y modernización de las artes y la arquitectura en el país como la Revista de Arquitectura, Nuestra Arquitectura, Ciclo, Canon, Ver y Estimar y nv nueva visión.

${ }^{11}$ Entre los textos publicados en esta etapa se encuentran Como concebir el Urbanismo ([1943]: 1959) y La ciudad del futuro ([1943]: 1962) ambos de Le Corbusier; La metrópoli en la vida moderna ([1943]: 1957) de Piggot, Chevalier y Neutra, conformando un bloque que podemos enmarcar dentro de los postulados del Urbanismo del "movimiento moderno". La biblioteca se completa con el texto de Henry Churchill La ciudad es su población ([1943]: 1962), Planeamiento Urbano ([1940]: 1959) de Thomas Sharp, Ciudades en evolución
}

(c) Urbana: Rev. Eletrônica Cent. Interdiscip. Estud. Cid. $\quad$ Campinas (SP) $\quad$ v.8, n.3 [14] $\quad$ p.08-29 ISSN 1982-0569 
íntegramente referidos al debate norteamericano, centrado en la ciudad y su proceso de metropolización, que incorpora lecturas desde la economía, la política y lo social como claves del proceso de transformación, en definitiva, una serie de textos con eje en la planificación como motor del cambio ${ }^{12}$.

En la Revista $A \& P$ el rol de J. E. Hardoy abarca desde su actividad como co-director y autor de artículos y reseñas ${ }^{13}$ asumiendo además las tareas de selección y difusión de los nuevos preceptos de la planificación en clave desarrollista. Así la presencia de los integrantes del IPRUL en la revista permite comprobar la aparición de nuevas temáticas vinculadas a una interpretación de la disciplina urbana: la planificación. Esta se presenta tanto en forma de artículos, como en lo relativo a las noticias y los comentarios de revistas internacionales, situación que a su vez posibilita una lectura de los intentos de consolidación de un espacio de difusión no sólo de los modelos e ideas sino, principalmente, de las actividades del instituto. De esta forma, la participación activa de los integrantes del IPRUL y sus referentes en las páginas de $A \& P$ expone los vínculos y cruces entre J. E. Hardoy, el IPRUL y $A \& P$. La multiplicidad de enfoques disciplinares permite consolidar desde la revista una perspectiva basada en una noción más abstracta y comprensiva de la planificación, donde los factores económicos, políticos y sociales son considerados elementos claves para el desarrollo físico. En particular los artículos enfatizan el reconocimiento de los fenómenos locales desde abordajes históricos, económicos, sociales y políticos para explicar la condición de subdesarrollo de estas latitudes, sumado a la identificación de procesos particulares que deben ser articulados con nuevas teorías pensadas "desde" y "para" estas realidades. De esta forma, los textos seleccionados por Hardoy en $A \& P$ inauguran el ciclo de la planificación regional y urbana en la Argentina.

de Patrick Geddes ([1915]: 1962) y La imagen de la ciudad ([1960]: 1960) de Kevin Lynch, el cual es traducido en el mismo año de su publicación original y se constituye como el texto fundantes del discurso del urban design en sede norteamericana.

12 Entre los textos publicados en esta segunda etapa se encuentran La ciudad en la historia: sus orígenes, transformaciones y perspectivas ([1961]: 1966) del norteamericano Lewis Mumford; El intelectual contra la ciudad: de Thomas Jefferson a Frank Lloyd Wright ([1962]: 1967) de Morton y Lucia White; El crecimiento urbano de Estados Unidos ([1962]:1968) de Constance McLaughlin Green; El enigma metropolitano ([1967]:1970), compilado por James Wilson y La lucha por el techo ([1964]: 1967), del urbanista norteamericano Charles Abrams.

13 Como autor Hardoy publica tres artículos en la revista. El primero en el año 1963 "Las ciudades precolombinas" presenta un avance de su tesis doctoral realizada en HARVARD. El segundo refiere a los procesos de transformación de las ideas y los modelos de la disciplina en sede internacional "La carta de Atenas y la declaración de Delos" en la sección Crónica del número 3-4. El último tema presentado por J. E. Hardoy hace referencia al diseño de nuevas ciudades a través del artículo "El planeamiento de una ciudad satélite de Buenos Aires" publicado en A\&P No 5 y 6 de 1965, realizado junto a Eduardo Aubone, Ana María Noguerol y Tristán Noguerol.

(c) Urbana: Rev. Eletrônica Cent. Interdiscip. Estud. Cid.

Campinas (SP)

v.8, n.3 [14]

p.08-29

ISSN 1982-0569 
A escala regional J. E. Hardoy se constituye como uno de los principales promotores del tema de la ciudad latinoamericana desde la actividad editorial en SIAP y luego, a partir de 1970, creando el órgano de difusión de la Comisión de Desarrollo Urbano y Regional (CDUYR) de CLACSO y su respectiva revista y editorial. Fue durante su presidencia en SIAP (1966-1970) que se crea "el programa editorial "Ediciones SIAP" con sus tres componentes: la Revista de la Sociedad Interamericana de Planificación, la publicación de libros, y el Boletín Correo Informativo SIAP (CAMACHO, 1993, pp. 103).

Los primeros veinte números publicados fueron dirigidos por el abogado colombiano Pedro Pablo Morcillo ${ }^{14}$, modificando su estructura organizativa en paralelo a los cambios de dirección de la institución ${ }^{15}$. La participación de actores relevantes del período (Carlos Matus, Luis Unkil, Aníbal Quijano, Paul Singer y J. E. Hardoy, entre otros) demuestra que la revista fue un espacio de concurrencia y debate de personalidades de todo el continente en los temas referidos a la planificación y el desarrollo de la ciudad y el territorio latinoamericano desde una perspectiva interdisciplinaria.

Sin embargo, la participación directa de J. E. Hardoy en la Revista SIAP se limita a la publicación de ciertos artículos vinculados principalmente a sus temas de indagación en el marco institucional del CEUR como el caso de "Mecanismos de regulación de la tierra urbana y sub-urbana en América del Sur"16 junto a Raúl Basaldúa y Oscar Moreno en el Vol. I N03 de 1967 o el artículo individual "El paisaje urbano en Sudamérica"17 en el año 1969 en el que formula un modelo futuro para la ciudad en América del Sur desde un

\footnotetext{
${ }^{14}$ Pedro Pablo Morcillo (abogado, educador, asesor) Nacido en Colombia. Doctor en Derecho y Ciencias Políticas por la Universidad Nacional de Colombia y Master en Planeamiento Urbano y Regional de la Universidad de Pennsylvania en Estados Unidos. Secretario de la Presidencia de la República de Colombia para la Administración Pública y Gerente de la estatal Empresa Colombiana de Minas. Docente en Economía, Arquitectura, Planificación e investigación Urbana.

${ }^{15}$ En los primeros catorce (14) números el equipo editor se completaba con la Secretaria Editorial, Judith de Campos; la Secretaría Administrativa, Rosalba Restrepo y el Comité Editorial Asesor integrado por John Friedmann, Aldo Ferrer, Ralph Gakenheimer, Helio Jaguaribe, Ricardo Jordán, Alberto Mendoza, Eduardo Montoulieu y Luis Vera; sumado a un conjunto de corresponsales nacionales. A partir del No15 (Septiembre 1970) la Secretaría Administrativa queda a cargo de María Cielo Puerta. Y es en el No17 (Marzo-Junio 1971), bajo la presidencia de SIAP del mexicano Cuauhtemoc Cárdenas que la dirección de la Revista pasa a manos de Germán Samper Gnecco, el Editor Asociado a cargo de Martha S. de Kaplan, sumado al Comité Asesor integrado por Carlos Cardona, Ramiro Cardona, Jorge Rivera Farfán, Pedro Pablo Morcillo y Jaime Valenzuela, sumado a los corresponsales de los diferentes países de la región. A partir de diciembre de 1974, la dirección de la Revista vuelve a cambiar, contando en este período con la dirección del argentino Alejandro Rofman, manteniendo el director asociado e incorporando un nuevo comité asesor internacional en el que participa Paul Singer, Walter Stohr, Pedro Pablo Morcillo, Carlos Matus, Joel Ballesté, Luis Unikel, Anibal Quijano y Alberto Harth Deneke. En los años posteriores se incorpora el argentino Javier Lindeiboim como director asociado, pasando a desempeñar este cargo desde 1975 a 1976.

${ }^{16}$ Este artículo forma parte de una investigación realizada en el marco del CEUR para "The Center for Housing, Building and Planning" de las Naciones Unidas.

${ }^{17}$ Este articulo fue publicado en la Revista SIAP Vol III, Número 11, Septiembre 1969.
}

(c) Urbana: Rev. Eletrônica Cent. Interdiscip. Estud. Cid.

Campinas (SP)

v.8, n.3 [14]

p.08-29

ISSN 1982-0569 
enfoque que incorpora la temática de la protección de recursos naturales, el uso de la técnica y la tecnología en la producción de las ciudades en estas latitudes.

La reforma urbana se presenta como uno de los eje de indagación entre 1970 y 1976 con la publicación de dos artículos: "Reforma Urbana en América Latina"18 junto a Oscar Moreno y "Un ensayo de interpretación del proceso de urbanización de América Latina"19 ${ }^{\prime 19}$, donde se observa posicionamiento frente al tema que involucra los procesos socio-productivos de la urbanización que permiten delinear las condiciones propuestas para lograr la "estabilidad" urbana en los países de América del Sur.

A partir de 1976, se reconoce un viraje de las temáticas publicadas por J. E. Hardoy en SIAP, centrándose principalmente en los temas del hábitat popular, la vivienda y las condiciones socio-ambientales. En esta línea los artículos "Comentarios de la Ley General de Asentamientos Humanos" ${ }^{\prime 20} \mathrm{O}$ "La vivienda de los pobres"21 permiten reflejar la reflexión y las preocupaciones que guiaran el trabajo de J. E. Hardoy en los siguientes años, marcando un giro temático frente a los años "desarrollistas".

Desde la perspectiva del proyecto institucional de SIAP, a partir de 1970 se incorpora en su estructura organizativa el Comité Editorial a cargo del programa "Libros Ediciones SIAP", que contaba con el financiamiento de la Fundación Ford y tuvo su sede en la ciudad de Buenos Aires ${ }^{22}$, contando con la presencia de J. E. Hardoy en la primera Comisión Editorial ${ }^{23}$.

En esta línea resulta posible rastrear los vínculos entre proyectos institucionales y proyectos editoriales que consolidaron la transformación disciplinar con eje en una perspectiva regional, social y económica desde América Latina. La difusión de textos claves, sumada a su propia producción le permitieron a J. E. Hardoy gestionar estos espacios editoriales (en diferentes grados), posibilitando un cruce entre experiencias locales y la consolidación de una visión regional que, pese a las diferencias, mantuvo líneas

${ }^{18}$ Este articulo forma parte de la recopilación de investigaciones ganadoras del Concurso Bienal "Premios SIAP 1972 ", en el contexto del noveno Congreso Interamericano de Planificación organizado en Bogotá. Publicado en la Revista SIAP Vol VII, Número 25, Marzo 1972.

${ }^{19}$ El artículo forma parte de la publicación "Food, population and emplyment: the impact of the Green revolution". Poleman y Freebairn editors. Praeger publishers, Ney Tork. 1973. Publicado en Revista SIAP, Vol. VII No 27 de septiembre de 1973

20 Publicado en Revista SIAP Vol. X No 37 de marzo de 1976

${ }^{21}$ Publicado en Revista SIAP Vol. X No 40 de diciembre de 1976

22 Entre 1970 y 1980 el programa editorial funciona en Buenos Aires. A partir de 1980 la sede se traslada a la ciudad de México, realizando las actividades directamente desde la Secretaría General de SIAP.

${ }^{23}$ Esta primera comisión estaba integrada además por Cuauhtemoc Cárdenas (Mex.), Ralph Gakenheimer (EE.UU), Alejandro Rofman (Arg), y José A. Silva Michelena (Ven.), y Martha S. de Kaplan (Arg.) como Editora Ejecutiva.

(c) Urbana: Rev. Eletrônica Cent. Interdiscip. Estud. Cid.

Campinas (SP)

v.8, n.3 [14]

p.08-29

ISSN 1982-0569 
articuladoras entre la investigación sobre ciudades precolombinas y la expansión urbana característica de los años cincuenta y sesenta.

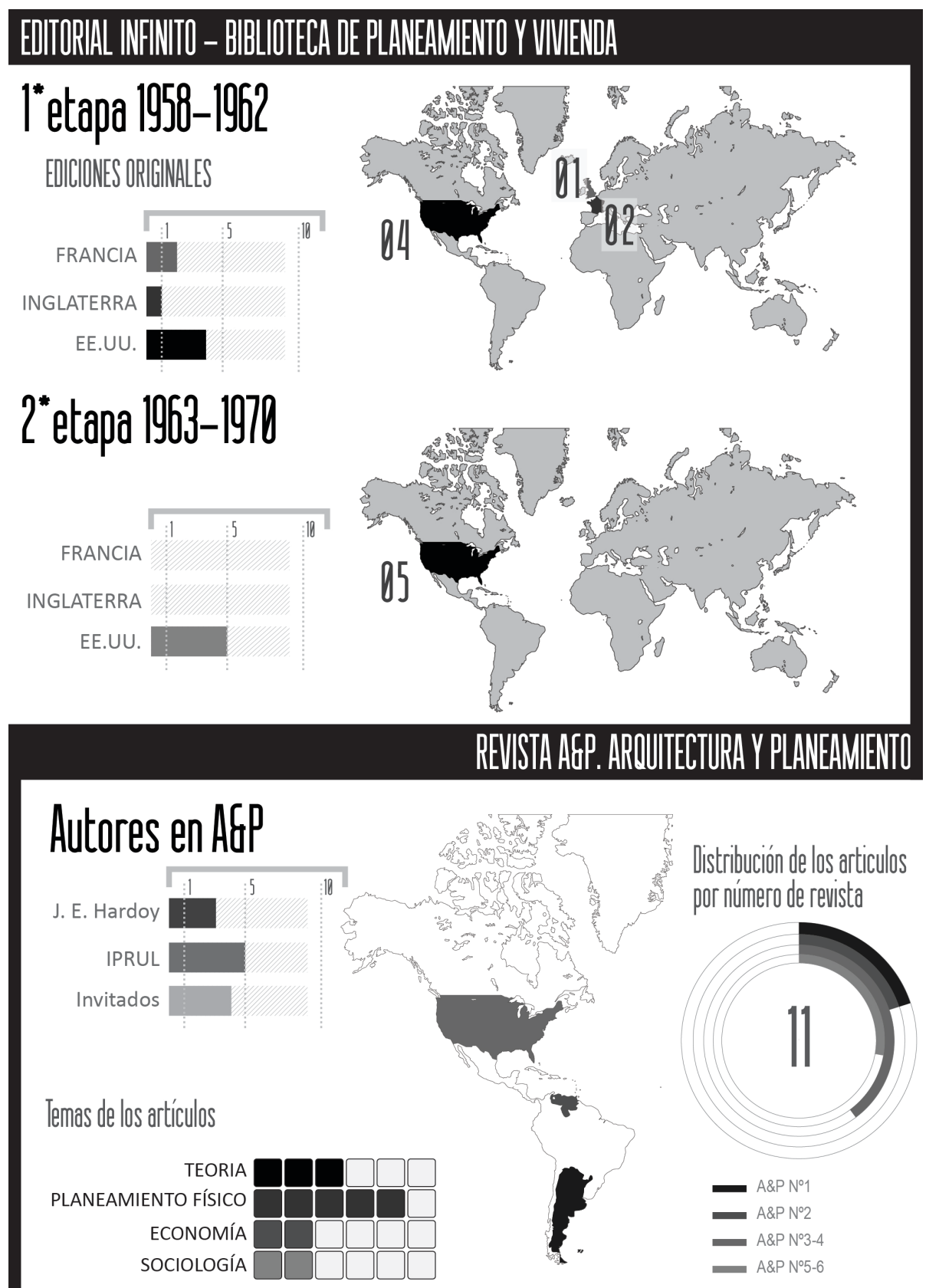

Figura 3 - Esquemas del recorrido editorial de J. E. Hardoy. Elaboración propia. 


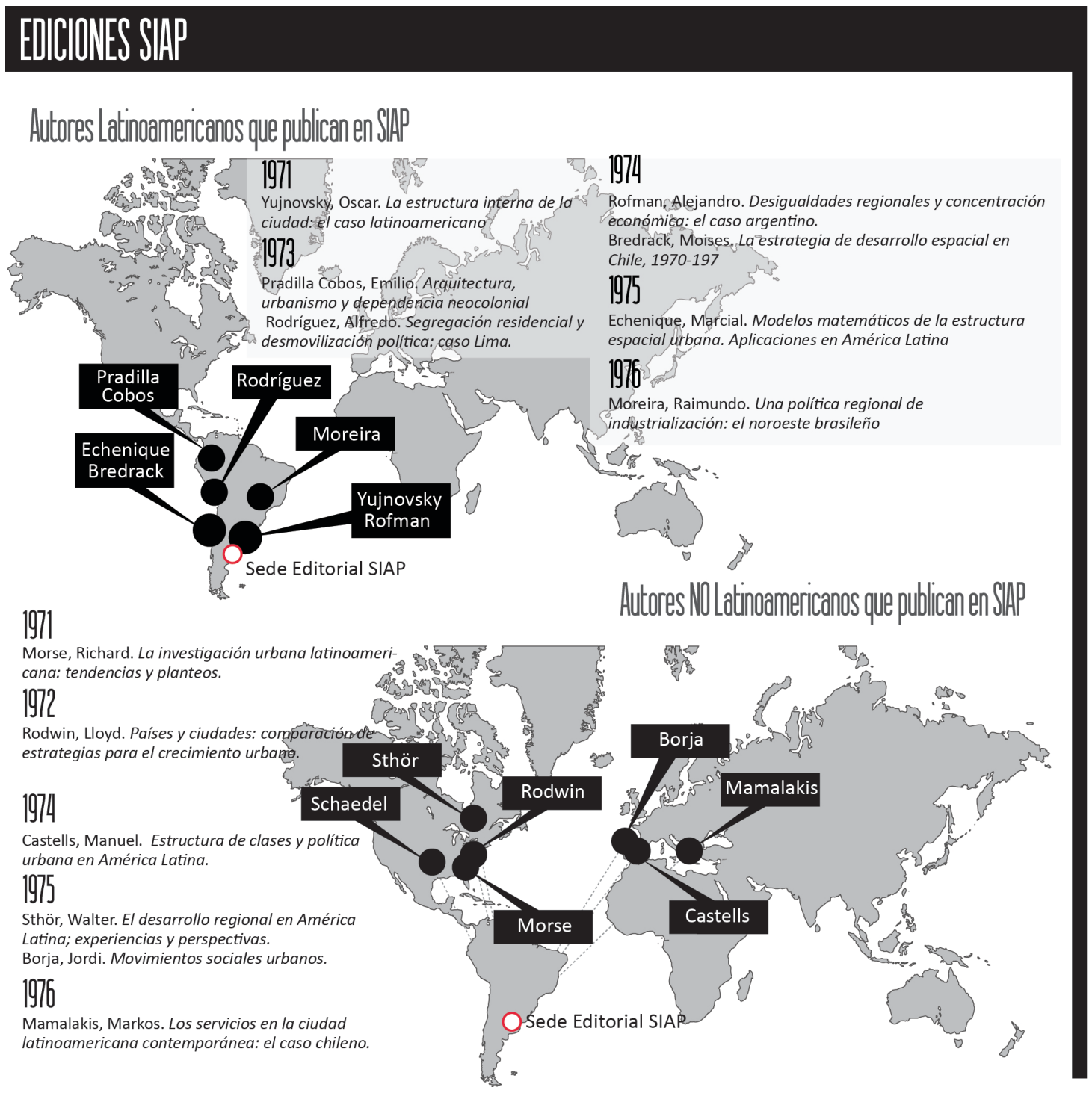

Figura 4 - Esquemas del recorrido editorial de J. E. Hardoy. Elaboración propia.

\section{Aproximaciones finales}

J. E. Hardoy fue un actor central en la transformación de la disciplina en la Argentina entre los años cincuenta y setenta. En su carácter de experto, pero sobre todo de promotor académico introdujo nuevas ideas y modelos para abordar los problemas de la ciudad y el territorio desde una perspectiva más comprehensiva vinculada a la noción de planificación como método de toma de decisiones tendientes al desarrollo y al equilibrio territorial en clave económica, donde la dimensión física es un índice y un campo subsidiario de actuación más que un fin en sí mismo.

Analizar su trayectoria desde la figura del experto, entendida como un nuevo tipo de profesional altamente calificado con un dominio de la técnica que se supone neutra y eficaz para la búsqueda del bien común, apto para integrarse a oficinas estatales,

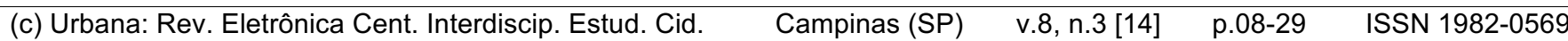


conformar equipos de trabajo y producir investigaciones y trabajos profesionales bajo la figura de la asistencia técnica al medio, posibilita identificar la riqueza del marco de referencias de J. E. Hardoy en el contexto de la Argentina pos-peronista; una trayectoria que reúne su actividad como arquitecto, urbanista y planificador (no sin contradicciones), pero que además se presentan tensionadas por fuerzas internas y externas según una lectura que refiere a momentos de conflicto en su tarea técnica y de investigación vinculadas a distintos períodos del debate disciplinar.

Pero la relevancia de este actor en el desarrollo de la disciplina urbana excede este rol de experto. Fue por su potencia como promotor académico, y su triple actividad como intelectual, gestor institucional y gestor editorial, que podemos plantear su relevancia en el ámbito del urbanismo en sentido amplio, argentino y latinoamericano.

Sostenemos que la trayectoria de J. E. Hardoy en el período (1953-1976) combinó la producción intelectual, la conformación y dirección de instituciones y centros de investigación, y la producción editorial, lo que le permitió posicionarse dentro de la disciplina como un productor de conocimientos y generador de redes académicas, sumado a la consolidación y legitimación de teorías y modelos a partir de la selección de autores y textos editados. Es más, tal como plantea Diego Pereyra para la categoría de empresario académico, J. E. Hardoy obtuvo fondos para investigación en el marco del desarrollo de sus instituciones, monopolizó recursos simbólicos y materiales, identificó rivales a fin de delimitar su liderazgo, reconoció oportunidades de investigación nacionales e internacionales, negoció con sponsors como el Estado, la Fundación Ford o Naciones Unidas y construyó redes personales y académicas que afianzaron su relevancia institucional en múltiples escalas, situación que mostró una superación de su rol como simple experto para avanzar sobre una construcción más dinámica de su trayectoria a partir de su consolidación como promotor académico.

La construcción del liderazgo de J. E. Hardoy, bajo la concepción de un promotor académico, permite articular su actividad institucional, sus aportes en el campo intelectual y su rol editorial, tanto en sede local, como en la identificación de su participación activa en la conformación de una red latinoamericana de planificadores regionales y urbanos.

En el contexto internacional reestructurado por la Guerra Fría y las relaciones panamericanas de la Alianza para el Progreso, se multiplicó el interés en los Estados Unidos por las teorías tendientes a modernizar y desarrollar al denominado Tercer Mundo donde Latinoamérica se constituyó en un laboratorio, profundizando los vínculos y dependencias con Norteamérica, no sólo en el campo económico, sino también en el intelectual. En este (c) Urbana: Rev. Eletrônica Cent. Interdiscip. Estud. Cid. $\quad$ Campinas (SP) $\quad$ v.8, n.3 [14] $\quad$ p.08-29 $\quad$ ISSN 1982-0569 
proceso, la actuación de J. E. Hardoy fue decisiva, a partir de que fue capaz de construir un nuevo campo de actuación para los profesionales locales bajo la impronta de la teoría de la modernización y la influencia de los economistas de la CEPAL que, en sus diferentes períodos, sostuvieron y difundieron la teoría desarrollista y se instituyeron como ejes dominantes en el campo intelectual de la época.

La explosión urbana latinoamericana, como emergente de disfuncionalidades económicas, políticas y sociales, se constituyó en un tema central de abordaje para los intelectuales locales que, desde diferentes disciplinas e instituciones, conformaron el "nuevo" mapa intelectual, académico y político del pensamiento social latinoamericano. Como afirma Adrian Gorelik, la masa de estudios sobre la ciudad (antropológicos, sociológicos, históricos y geográficos) que se elaboran en este periodo no tiene precedentes y desde esta producción es posible reconstruir simultáneamente tanto el debate transnacional, como las tensiones políticas, culturales y sociales latinoamericanas vinculadas al proceso central de "explosión urbana". J. E. Hardoy contribuyó a reconocer el proceso de urbanización como eje articulador de los estudios interdisciplinarios fomentados por la Comisión Económica para América Latina (CEPAL) y la Sociedad Interamericana de Planificación (SIAP) y como motor para la creación de institutos de investigación en los países latinoamericanos, como por ejemplo el Centro de Estudios para el Desarrollo (CENDES, 1961) en Venezuela y el Comité Interdisciplinario de Investigación y Enseñanza del Planeamiento y del Desarrollo Urbano (CIDU, 1965) en Chile, en consonancia con el IPRUL (1961-1965) y posteriormente el CEUR (1965-1976) en la Argentina. La simultaneidad en la creación de estos institutos muestra, por un lado, la sintonía de J. E. Hardoy con los debates continentales del período y, por el otro, la importancia otorgada a los temas urbanos y regionales dentro de las agendas locales y particularmente a nivel latinoamericano, contando en todos los casos con la cooperación financiera y técnica de instituciones públicas y privadas, nacionales e internacionales.

La construcción de la categoría de promotor académico cristaliza un ciclo de la producción de J. E. Hardoy signado por el debate sobre la ciudad latinoamericana, su historia y sus temas/problemas desde una perspectiva interdisciplinaria y en sintonía con los debates y las instituciones nacionales y supranacionales. 


\section{REFERENCIAS}

ALMANDOZ, Arturo. Entre libros de historia urbana. Caracas: Ediciones Equinoccio, 2008.

------ "Urban planning and historiography in Latin America", Londres: Progress in planning N065, Pergamon, 2006

CAMACHO, Luis Eduardo. El paso de Jorge Enrique Hardoy por la presidencia de SIAP. Buenos Aires: Revista Medio Ambiente y Urbanización N045, 1993.

CAMACHO, Luis Eduardo. Sociedad Interamericana de Planificación, SIAP 50 años Vida institucional y programática". Bogotá: Revista Bitácora Urbano Territorial Vol. 11, No 1, 2007.

GORELIK, Adrian. La producción de la 'ciudad latinoamericana. San Pablo: Tempo Social. Revista de Sociología da USP, Volumen 17, No1, 2005

GUTIÉRREZ, Ramón. "Jorge Enrique Hardoy: su aporte a la historia urbana de América Latina". Santiago de Chile: Revista EURE Vol. XXI, N062, 1995 INSTITUTO TORCUATO DI TELLA. Memoria y balance 1965. Buenos Aires: ITDT, 1967. Memoria y balance 1967. Buenos Aires: ITDT, 1967.

KAPLAN, Marcos. "Prólogo". En HARDOY, J. E. Las ciudades en América Latina: seis ensayos sobre la urbanización contemporánea. Buenos Aires: Editorial Paidós, 1972 NEIBURG, Federico y PLOTKIN, Mariano (comp). Intelectuales expertos. La constitución del conocimiento social en la Argentina. Buenos Aires: Paidós, 2004. PEREYRA, Diego. Los científicos sociales como empresarios académicos. El caso de Gino Germani, El desarrollo de las ciencias sociales. Tradiciones, actores e instituciones en Argentina, Chile, México y América Central, San José de Costa Rica: Serie Cuadernos de Ciencias Sociales, FLACSO, 2010.

VILLAR, Claudia. El pensamiento del experto como objeto de estudio. La Plata: Revista de Filosofía y Teoría Política, N031-32, 1996. 